\title{
QUANTITATIVE MRI CHARACTERIZATION OF BRAIN ABNORMALITIES IN DE NOVO PARKINSONIAN PATIENTS
}

\author{
Verónica Muñoz Ramírez ${ }^{\star \dagger} \quad$ Florence Forbes ${ }^{\dagger} \quad$ Julyan Arbel ${ }^{\dagger} \quad$ Alexis Arnaud ${ }^{\dagger} \quad$ Michel Dojat ${ }^{\star}$ \\ * Grenoble Institut des Neurosciences, Inserm U1216, Univ. Grenoble Alpes, 38000 Grenoble, France \\ ${ }^{\dagger}$ Univ. Grenoble Alpes, Inria, CNRS, Grenoble INP, LJK, 38000 Grenoble, France
}

\begin{abstract}
Currently there is an important delay between the onset of Parkinson's disease and its diagnosis. The detection of changes in physical properties of brain structures may help to detect the disease earlier. In this work, we propose to take advantage of the informative features provided by quantitative MRI to construct statistical models representing healthy brain tissues. This allows us to detect atypical values for these features in the brain of Parkinsonian patients. We introduce mixture models to capture the non-standard shape of the data multivariate distribution. Promising preliminary results demonstrate the potential of our approach in discriminating patients from controls and revealing the subcortical structures the most impacted by the disease.
\end{abstract}

Index Terms - Brain, Biomarker, Statistical mixture models

\section{INTRODUCTION}

Parkinson's Disease (PD) is a complex neurodegenerative disease that can be divided into two stages. First, a prodromal phase lasting from a few years to several decades where dopaminergic neurons are lost progressively, affecting mainly subcortical brain structures. Then, a clinical phase involving cortical networks and marked by PD motor symptoms, such as gait impairment, rigidity and resting tremor. It would be of great importance, with respect to research and treatment, to identify biomarkers that may yield early PD diagnosis. Magnetic Resonance Imaging (MRI) is a non-invasive imaging technique useful for the detection, characterization and monitoring of neurological changes in de novo, i.e. just diagnosed, PD patients. Several innovative quantitative MRI techniques have been developed to measure meaningful physical or chemical brain properties [1]. The combination of measures from multiple MRI modalities may provide a complete picture of functional and structural changes caused by PD [2].

In the quest to find biomarkers that accurately differentiate PD patients from healthy subjects, we propose an abnormality detection approach that identifies unusual patterns in

VMR is supported by a grant from NeuroCoG IDEX UGA in the framework of the Investissements dávenir program (ANR-15-IDEX-02). multi-parametric MRI data. For this purpose, we fit a mixture model to data from healthy volunteers (reference model), then compare to de novo PD data to search for outliers, i.e. possible abnormalities, in different brain regions, to which we fit another mixture model (atypical model) to represent the potentially complex distribution of abnormal tissues.

Mixture models are probabilistic models that represent subpopulations within an overall population. They do not require prior knowledge of which subpopulation a data point belongs to, allowing the model to learn automatically the subpopulations in an unsupervised manner.

In our study, the reference model is built assuming that healthy subjects present a set of distinct healthy tissues characterized by different quantitative characteristics. These classes of characteristics are captured using a mixture model with an appropriate number of components, whose number is determined automatically from the data. Abnormal regions in PD patients are then detected as those with a too low logdensity score in the reference model. The detected abnormal regions are in turn used to build another mixture that defines a new model for atypical characteristics.

\section{QUANTITATIVE MRI DATA}

Quantitative MR data are considered, in particular, to be as independent as possible of the MRI scanner or the study center [1]. The scanner is no longer considered only as a camera but as a means of measurement. In this paper, we consider Diffusion imaging (DI) and Perfusion Imaging (PI). Through DI, mean diffusivity [MD] describes the overall displacement of water molecules and fractional anisotropy [FA] indicates the orientation of diffusion. Although there are some inconsistent findings regarding diffusivity in PD patients, DI has been helpful to discriminate PD from other syndromes [3, 4]. Moreover, with the development of arterial spin labelling (ASL), brain perfusion can be assessed non-invasively. Cerebral blood flow (CBF) has been reported to be decreased in the cortex of PD patients and either preserved or decreased in their basal nuclei $[5,6]$.

To explore the feasibility of our method described in Section 3, we extracted FA and MD parameter maps from DI, and $\mathrm{CBF}$ maps from PI, for three healthy subjects (C1-C3) 
(Age: $\{28,40,50\} ; 2$ Males) and nine de novo PD patients (P1-P9) (Age $\in[36,66]$; H\&Y score $\leq 2$; 7 Males). All images were acquired on a Philips $3 \mathrm{~T}$ scanner. For each individual, the computed maps were coregistered and resliced $(1 \mathrm{~mm}$ isotropic) with SPM12 to obtain, for each voxel $v$, three corresponding feature values $y_{v}=\left(\mathrm{FA}_{v}, \mathrm{MD}_{v}, \mathrm{CBF}_{v}\right)$.

In this first attempt, we have searched for outliers in the subcortical brain structures where changes were most likely to take place in de novo PD patients, namely the substantia nigra (SN), the red nucleus (RN), the subthalamic nucleus (STN), the Globus Pallidus interna and externa (GPi, GPe), the thalamus, the putamen and the caudate nucleus. To obtain a mask of these Regions of Interest (ROIs), we performed a non-linear deformation of the MNI PD25 atlas [7], specifically designed for PD patient exploration. In addition, we considered the superior colliculus (SC) and the inferior colliculus (IC), where we recently found functional deficits [8]. The results of this experiment are summarized in Section 4.

\section{ABNORMALITY DETECTION VIA MIXTURE MODELS}

To eliminate possible redundant information, we perform a Principal Component Analysis (PCA) on the three maps (CBF, FA and MD) for our healthy subjects. Each parameter contributes equally to the information of explained variance $(38 \%, 33 \%, 29 \%)$ on axes $(1,2,3)$ and all parameters are then considered in the statistical analysis. A following step is then to decide on an appropriate model that best accounts for the data distribution. Although Gaussian distributions are the most widely used statistical distributions for their tractability and representation power, they are constrained by elliptical shapes. Mixtures of Gaussians can help in modelling richer distributional shapes but they are still not appropriate when the data present elongated and strongly non-elliptical subgroups. As an alternative, we consider a richer family of distributions based on multiple scaled $t$-distribution (MSD) mixtures. These heavy-tailed distributions are endowed with variable marginal amounts of tail-weight and their mixtures have been showed to provide an efficient alternative to Gaussian mixtures $[9,10]$. In particular, their ability to model over-dispersed values is illustrated in Figure 1.

The tridimensional measures (FA, MD, CBF) are separated into healthy and patient datasets,

$$
Y_{H}=\left\{y_{v}, v \in V_{H}\right\} \quad \text { and } Y_{P}=\left\{y_{v}, v \in V_{P}\right\},
$$

where $V_{H}$ and $V_{P}$ respectively represent the voxels belonging to healthy volunteers and to patients. By fitting an MSD mixture to the healthy data $Y_{H}$, we build a reference model density $f_{H}$ that describes healthy tissues as follows:

$$
f_{H}(y \mid \boldsymbol{\pi}, \boldsymbol{\theta})=\sum_{k=1}^{K_{H}} \pi_{k} \operatorname{MSD}\left(y ; \theta_{k}\right),
$$

where $K_{H}$ is the number of mixture components, and each component is characterized by a proportion $\pi_{k}$ and an MSD

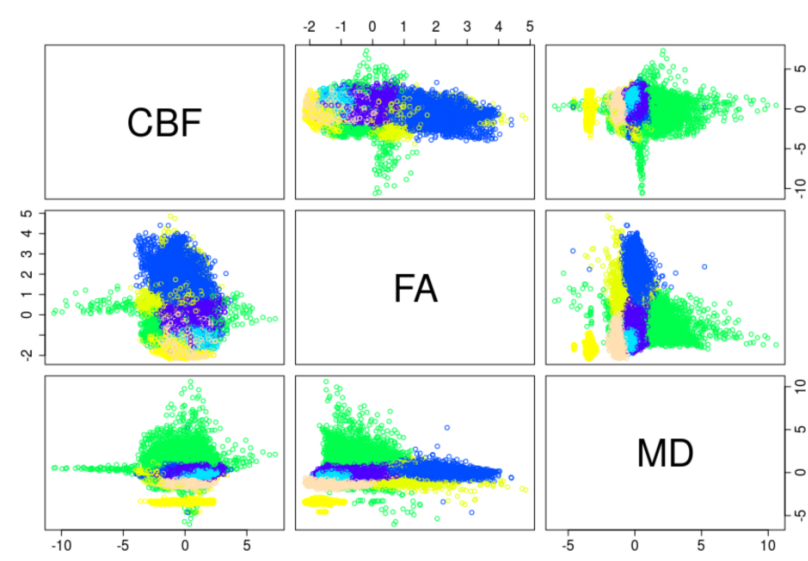

Fig. 1. Bivariate marginal distributions of our three parameter maps (CBF, FA, MD). The colors represent the different classes assigned by the MSD mixture reference model (2).

parameter $\theta_{k}$. The optimal number of components $K_{H}$ is chosen using the Bayesian Information Criterium (BIC). The Expectation-Maximization algorithm (EM) [9] is used to obtain the best fit of the MSD mixture on $Y_{H}$ : it jointly estimates the weights $\boldsymbol{\pi}$ and the parameters $\boldsymbol{\theta}$ by reaching a (local) maximum of the model log-likelihood.

The log-density (or log-score) of each voxel (in $V_{H}$ and $V_{P}$ ) with respect to $f_{H},\left\{\log f_{H}\left(y_{v}\right), v \in V_{H} \cup V_{P}\right\}$, can then be used to compute a threshold to detect abnormal voxels. The log-score can be considered as a measure of proximity of one voxel $v$ (associated to value $y_{v}$ ) to the reference healthy model (represented by $f_{H}$ ). The rational is that voxels from healthy subjects are more likely to have a high logscore while pathological voxels may not be well explained by the reference model and consequently have a lower log-score. A threshold can be computed in a data-driven way by fitting a 2-component mixture model on the log-scores, defining the threshold at the intersection of the two distributions. This separation into high and low score groups would correspond to a certain false positive error rate (FPR) that can be determined as proposed in [10]. We choose another option which consists of deciding on an acceptable FPR $\alpha$ and determining the corresponding threshold $\tau_{\alpha}$ : the probability the $\log$-score is smaller than $\tau_{\alpha}$, although the log-score is that of an healthy voxel, is $\alpha$. In other words, $\tau_{\alpha}$ is the value such that

$$
P\left(\log \left(f_{H}(Y)\right)<\tau_{\alpha}\right)=\alpha,
$$

when $Y$ is a random variable following the $f_{h}$ reference model distribution. In practice, while $f_{H}$ is known explicitly, the probability distribution of $\log \left(f_{H}(Y)\right)$ is not. However, it is easy to simulate this distribution so that $\tau_{\alpha}$ can be computed using empirical quantiles (Figure 2).

All the voxels whose $\log$-score is below $\tau_{\alpha}$ are then labeled as abnormal and the corresponding measures provide a 
set of parameters that are referred to as the abnormal data set

$$
Y_{A}=\left\{y_{v}, v \in V_{H} \cup V_{P} \text {, s.t. } \log f_{H}\left(y_{v}\right)<\tau_{\alpha}\right\} .
$$

An abnormality model denoted by $f_{A}$ is then constructed following the same procedure as for the reference model:

$$
f_{A}(y \mid \boldsymbol{\mu}, \boldsymbol{\phi})=\sum_{k=1}^{K_{A}} \mu_{k} \operatorname{MSD}\left(y ; \phi_{k}\right) .
$$

This abnormality model is used to account for the fact that voxels detected as abnormal may belong to different abnormality classes, with different physiological characteristics. Typically, the above formula indicates that among the $Y_{A}$ set there are $K_{A}$ different groups. Theses groups can be used to build a signature $\rho^{s}$ of each subject $s$ by determining the proportion of voxels that are assigned to each of the $K_{A}$ classes:

$$
\rho^{s}=\left(p_{1}^{s}, \ldots, p_{K_{A}}^{s}\right) .
$$

Figure 3 provides an example of such signatures. The top plot illustrates that abnormal voxels can be detected in healthy subject although in a much lower proportion that in PD subjects. In addition the abnormality pattern in healthy subjects is usually different and may be distinguished from PD patterns. A model of this difference can be quantified using standard discriminant analysis models and the known status of each subject (control or patient). As explained in [10], we can make use of this classification information and of additional spatial information to refine abnormality detection. Notably, clusters of less than 4 atypical voxels are not selected. Figure 3-bottom shows the effect of such a post-processing.

\section{RESULTS}

For control data, the highest BIC score for the MSD mixture reference model (2) is obtained for $K_{H}=7$ components. It is critical that the reference model correctly characterizes the physiological properties of brain structures while encompassing the individual variability. Interestingly, while individual differences emerge, a spatial symmetry is observed over the two brain hemispheres, when looking at individual brain slices annotated with the reference classification. The proportion of each class in the control subjects provides an insight into these differences, see Figure 2(A).

To separate atypical from healthy voxels we choose a FPR of $\alpha=5 \%$. Figure 2(B) shows the density of the log-scores computed for all voxels with respect to the reference model. Voxels considered as abnormal are located left to the vertical red line representing the threshold $\tau_{\alpha} .49196$ voxels are labelled as abnormal (i.e. $20.4 \%$ of the set of voxels). Note that the majority of these abnormal voxels belong to PD patients, however, but some are detected in the controls. This is expected for two reasons. First, because each healthy individual is unique and can bring some non-pathological extreme values, just like for a patient, the physiological measures are not

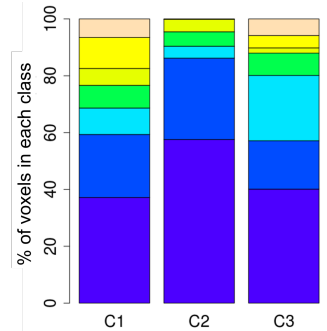

(A)

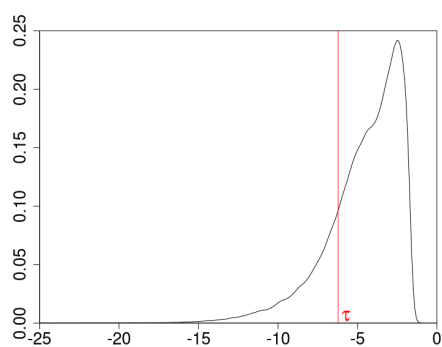

(B)
Fig. 2. Panel (A): proportion of each of the $K_{H}=7$ classes for controls $\mathrm{C} 1, \mathrm{C} 2$ and $\mathrm{C} 3$ given by the reference model $f_{H}$. Panel (B): density of the log-scores computed for all voxels (controls and patients) with respect to $f_{H}$. The red line represents the abnormality threshold $\tau_{\alpha}$.
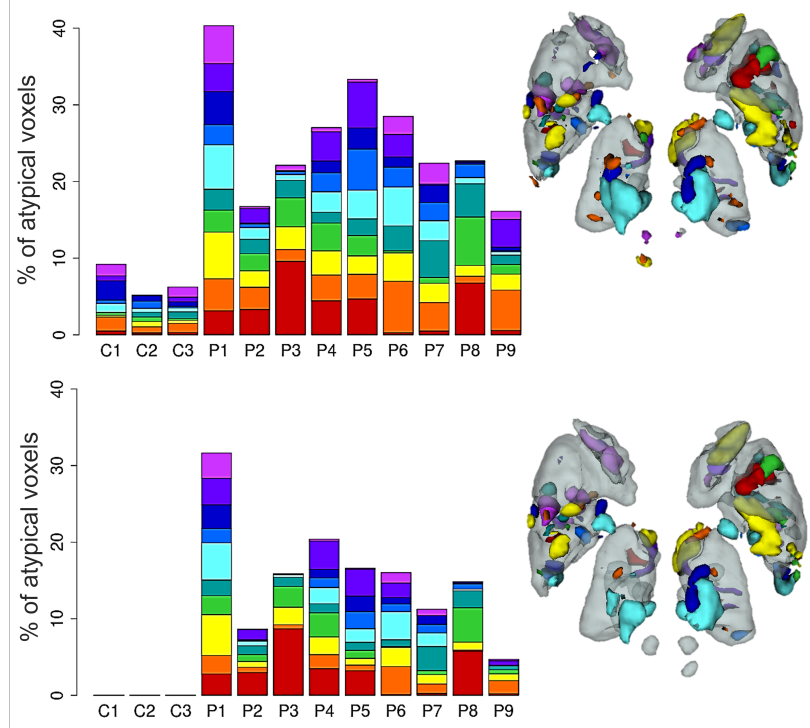

Fig. 3. Subjects signatures derived from $f_{A}$ before (top) and after post-processing (bottom). Each color represents one the $K_{A}=10$ abnormal classes. Their localization in the brain is illustrated for PD patient P1 (right).

all abnormal; and second, because we have admitted a nonzero FPR.

The abnormal model is best defined by $K_{A}=10$ classes according to BIC score. Figure 3 displays the abnormal signatures of all subjects before and after post-processing. After post-processing only 28377 voxels, i.e $11.8 \%$ of all voxels, remain labelled as abnormal. For Controls, no more voxels are detected as abnormal. The largest numbers of abnormal voxels are detected for patients $\mathrm{P} 1$ and $\mathrm{P} 4$. This is coherent with the corresponding additional functional MRI data obtained for these two subjects, which show alteration in visual information processing in their subcortical structures[8].

Table 1 indicates the localization for each PD patient of 
abnormal voxels with regard to the corresponding subcortical structures. We computed the percentage of each ROI that was detected as abnormal. In accordance with the literature, with found that the substantia nigra $(\mathrm{SN})$, the red nucleus (RN) and the globus pallidus (GP) were the most impacted by the pathology. Indeed,the degeneration of dopaminergic neurons in the SN pars compacta and the subsequent denervation of the dorsal striatum are at the origin of PD [11]. Abnormal signatures found in SN, GP and Cau are also in line with the reduced degree of myelination found in these regions for $\mathrm{PD}$ patients $[12,5]$. Moreover, $\mathrm{SN}$ and RN show an augmented FA [3] and there is evidence of a decrease in structural connectivity between SN, ipsilateral putamen and thalamus [13];

\begin{tabular}{|c|c|c|c|c|c|c|c|c|c|}
\hline \% & P1 & P2 & P3 & P4 & P5 & P6 & P7 & P8 & P9 \\
\hline SN & 71 & 19 & 18 & 64 & 10 & 49 & 60 & 29 & 7 \\
\hline RN & 48 & 1 & 0 & 7 & 41 & 31 & 27 & 24 & 0 \\
\hline STN & 22 & 2 & 0 & 45 & 0 & 11 & 12 & 0 & 0 \\
\hline GPe & 49 & 4 & 9 & 24 & 29 & 21 & 19 & 5 & 10 \\
\hline GPi & 63 & 4 & 1 & 21 & 11 & 39 & 24 & 0 & 22 \\
\hline Th & 20 & 10 & 32 & 11 & 12 & 16 & 5 & 32 & 3 \\
\hline Put & 30 & 11 & 7 & 25 & 20 & 5 & 7 & 4 & 3 \\
\hline Cau & 27 & 1 & 4 & 14 & 28 & 13 & 15 & 5 & 0 \\
\hline SC & 0 & 0 & 13 & 0 & 0 & 22 & 16 & 0 & 0 \\
\hline IC & 33 & 0 & 0 & 0 & 0 & 18 & 58 & 13 & 0 \\
\hline
\end{tabular}

Table 1. Percentages of abnormal voxels after postprocessing in each patient subcortical structure.

\section{CONCLUSION AND PERSPECTIVES}

We presented a method for the detection of abnormalities in de novo PD patients. We demonstrated its potential on a small cohort of three controls and nine PD patients. Clearly, more subjects are needed to bring out robust biomarkers of PD. However, these preliminary results show that the application of mixture models of relevant distributions is informative and promising to correctly discriminate the pathology. In addition, our method, in contrast to supervised machine learning techniques, does not rely on a large set of annotated data, which are difficult to obtain in medical contexts. Moreover, it relies on interpretable statistical tools that can be tuned and compared, providing a model of physical properties alterations. Last but not least, it does not require groundtruth comparison, making it a valuable tool for the exploration of any physiological changes. Future work will extend our method to the study of the entire brain on large cohorts and on the inclusion of new physiological measures to fully exploit the potential of multi-parametric quantitative MRI.

\section{REFERENCES}

[1] P. Tofts, Quantitative MRI of the brain, Wiley, 1st edition, 2005.
[2] F. D. B. Bowman \& al., "Multimodal imaging signatures of Parkinson's disease," Frontiers in Neuroscience, vol. 10, pp. 1-11, 2016.

[3] S. T. Schwarz \& al., "Diffusion tensor imaging of nigral degeneration in Parkinson's disease: A region-ofinterest and voxel-based study at $3 \mathrm{~T}$ and systematic review with meta-analysis," NeuroImage: Clinical, vol. 3, pp. 481-488, 2013.

[4] G. Du \& al., "Combined R2* and Diffusion Tensor Imaging Changes in the Substantia Nigra in Parkinson's Disease," Movement Disorders, vol. 26, no. 9, pp. 162732, 2011.

[5] N. Pyatigorskaya \& al., "A review of the use of magnetic resonance imaging in Parkinson's disease," Therapeutic Advances in Neurological Disorders, vol. 7, no. 4, pp. 206-220, 2014.

[6] T. R. Barber \& al., "Neuroimaging in pre-motor Parkinson's disease," NeuroImage: Clinical, vol. 15, pp. 215227, jan 2017.

[7] Y. Xiao \& al., "Multi-contrast unbiased MRI atlas of a Parkinson's disease population," International Journal of Computer Assisted Radiology and Surgery, vol. 10, no. 3, pp. 329-341, 2015.

[8] E. Bellot \& al., "First steps of visual processing are altered in de novo Parkinson's disease patients," in IAPRD Conf, Lyon, FR, 2018.

[9] F. Forbes and D. Wraith, "A new family of multivariate heavy-tailed distributions with variable marginal amounts of tailweight: application to robust clustering," Statistics and Computing, vol. 24, no. 6, pp. 971-984, 2014.

[10] A. Arnaud \& al., "Fully Automatic Lesion Localization and Characterization: Application to Brain Tumors Using Multiparametric Quantitative MRI Data," IEEE Transactions on Medical Imaging, vol. 37, no. 7, pp. 1678-1689, 2018.

[11] M. Politis, "Neuroimaging in Parkinson disease: from research setting to clinical practice," Nature, vol. 10, pp. 708-722, 2014.

[12] P. Péran \& al., "Magnetic resonance imaging markers of Parkinson's disease nigrostriatal signature," Brain, vol. 133, no. 11, pp. 3423-33, 2010.

[13] L. Griffanti \& al., "Exploring variability in basal ganglia connectivity with functional MRI in healthy aging," Brain Imaging and Behavior, pp. 1-6, 2018. 